\title{
Anesthesia for carotid endarterectomy: where do we stand at present?
}

BY DRAGANA UNIC-STOJANOVIC, DJORDJE RADAK, TAMARA GOJKOVIC, PREDRAG MATIC, LJILJANA RANKOVIĆ, MIOMIR JOVIC

\section{Abstract}

Carotid endarterectomy (CEA) is a surgical procedure performed to reduce the incidence of embolic and thrombotic stroke. Although only a preventive procedure, CEA carries the risk of perioperative complications. There is constant searching for an optimal anesthetic technique. There are pros and cons for both anesthetic techniques used: regional (RA) and general anesthesia (GA). A large number of studies have compared RA and GA techniques in CEA surgery patients. The primary outcome was the proportion of patients with stroke, myocardial infarction, or death. However, neither the GALA trial nor the pooled analysis was adequately powered to reliably detect an effect of type of anesthesia on mortality. It may therefore be appropriate to consider other additional parameters (stress response, incidence of postoperative delirium and cognitive impairment, functional recovery, total surgery time, intensive care unit requirement, hospital stay, hospital costs and patients satisfaction) when comparing the outcomes of the two techniques.

Although, the debate continues as to whether regional anesthesia or general anesthesia is safer, the choice of anesthetic technique is a complex decision and surgical teams should be able to offer both RA and 
GA. The individual approach is the ideal choice and should be determined at the discretion of the surgeon, anesthetist and patient depending on the clinical situation and own preferences.

Key words: surgery, carotid endarterectomy, anesthesia, general, regional, outcome

\section{Introduction}

Carotid artery disease presents a local manifestation of systemic disease. Patients with carotid artery disease may have been asymptomatic or have already suffered a transient ischemic attack or stroke, which is the most common cause of neurological disability in adults in the developed world.

Carotid endarterectomy (CEA) is a surgical procedure performed to restore carotid blood flow and to reduce the incidence of embolic and thrombotic stroke. In relation to postoperative outcome, the indications for CEA in symptomatic and asymptomatic patients are clearly defined in recently published guidelines for carotid disease treatment. $(1,2)$ CEA is absolutely indicated in symptomatic patients with internal carotid artery (ICA) stenosis $>50 \%$, if perioperative stroke/death rate is $<6 \%$. $(1,2)$ CEA is recommended in asymptomatic men, younger than 75 years with ICA stenosis $70 \%$ to $99 \%$, if the risk associated with surgery is less than $3 \%$. $(1,2)$ Asymptomatic women with carotid stenosis have less benefit from CEA than men and surgery may be reasonable in well-informed, young, fit individuals. $(1,2)$

Although only a preventive procedure, CEA carries the risk of perioperative complications: neurological and cardiac. The major complications of CEA are intra- and postoperative stroke, myocardial infarction (MI), and death. The incidence of perioperative stroke during CEA is approximately 2.3\%. $(3,4)$ The incidence of perioperative MI is $<2 \%$. $(4,5)$ The overall mortality for CEA was reported to be $1.3 \%$ to $1.8 \%$. $(4,6,7)$

Patients undergoing CEA are anesthetized with the following techniques (4): general anesthesia (GA) with inhaled or total intravenous anesthesia, and regional anesthesia (RA) with a deep or superficial block or both or 
cervical epidural anesthesia. There is a widespread view amongst anesthetists, surgeons, patients and other healthcare professionals that major surgery is inherently safer, if it can be performed under local (LA) or regional anesthesia rather than GA, particularly if the patient has multiple co-morbidities. CEA is a procedure, where such views are often polarized, so that at some centers, GA is not being offered as an option at all. However, is there any real evidence to support such a stance, either for this or other procedures in the medically fit or unfit patient?

\section{RA: pros and cons}

Almost all practitioners agree that there is a need to monitor cerebral blood flow (CBF) during CEA, particularly during the period of arterial cross clamping. Regional anesthesia is the only technique that will correctly identify all patients requiring shunt insertion for carotid artery cross clamping. (8) Consequently, fewer shunts are used. (8) This is important, because although shunts should protect the brain from stroke that results from low cerebral blood flow during carotid clamping, shunts can damage the arterial wall causing embolism to the brain. Other possible benefits of RA include the avoidance of tracheal intubation in patients with chronic obstructive lung disease, and avoidance of negative inotropic anesthetic agents in patients with limited cardiac reserves, avoidance of some adverse consequences of GA and preserved cerebral autoregulation of perfusion. $(4,9)$ RA also provides better pain control and improves immediate postoperative neurologic assessment, and earlier mobilization leading to reduced hospital stay. Also, RA allows arterial closure at 'normal' arterial pressure with possible reduced risk of postoperative hematoma.

On the other hand, the following patients would not be considered candidates for a regional block: patients who are unable to remain still or follow directions; patients with joint problems, tremors, or neurologic disorders; anxious, agitated, or claustrophobic patients, as well as patients with a short, fat neck. (4) Also, the patient should be alert enough to do awake neurological testing during carotid clamping, but short periods of deep sedation might be needed if the patient becomes restless or uncomfortable, particularly towards the end of the procedure. 
The operation under RA may be more hurried and technically more difficult which may increase the risk of a poor result from surgery. More hurried surgery during RA might result in arterial trauma, and subsequent thrombosis and embolism. Limited access to the airway during carotid surgery with the head turned to one side makes tracheal intubation technically difficult and associated with hemodynamic instability and possible cerebral hypoperfusion. $(4,10)$

Also, not all patients are psychologically able to tolerate the procedure while awake, and around 10\% of patients refuse the surgery under RA. (11) Also, some surgeons find performing the surgery under RA stressful. RA cannot provide potential pharmacologic brain protection with anesthetics.

Each regional technique has its complications, including: inadequate anesthesia, subarachnoid/epidural/intravascular injection, hematoma, phrenic nerve palsy, recurrent laryngeal nerve block, vagus nerve block and Horner's syndrome. Pandit et al. (12) compared the incidence of complications associated with the use of a deep block (whether as a sole technique or as part of a combined block) versus the use of superficial or intermediate block alone, and showed there were no complications arising from block placement in superficial block (o\%) when compared with $0.25 \%$ in deep block ( $P=0.006$; odds ratio 2.13; relative risk 2.12). However, the absolute incidence of block-related complications is low in both groups. Surprisingly, they found that the deep block is about five times more likely to "fail" and to be converted to GA compared to the superficial block. There were $0.39 \%$ conversions to GA in superficial when compared to $2.08 \%$ in deep block $(P<0.0001$; odds ratio 5.35; relative risk 5.26). The reasons for conversion to general anesthesia after placement of the block included: any block-related serious complication, failure of adequate analgesia (i.e. block failure); lack of patient cooperation after insertion of the block; or extreme patient anxiety. If the conversion rate to GA reflects success, then performing a superficial block is better than a deep block. This is not all. Superficial block is as effective as a deep or combined block if the amount of local anesthetic supplementation by the surgeon is used as a measure of block efficacy, (9) and other measures of block effectiveness (pain scores, postoperative 
analgesia), which were also not different between the two types of regional anesthesia. (13)

\section{GA: pros and cons}

The main advantages of GA are a safe airway throughout the procedure with controlled ventilation and arterial carbon dioxide concentrations.

(4) Anesthesia can be maintained using inhalational or i.v. agents. There are theoretical neuroprotective effects of GA agents (inhalational and i.v.). $(4,10)$ and some hemodynamic advantage of propofol anesthesia during carotid clamping. (14) However, currently, there is no outcomebased evidence favoring any particular general anesthetic agent during carotid clamping. (4)

The disadvantages of GA include the usual complications of GA (major airway problems, headache and sore throat) and the residual effects of GA in the early postoperative period that can mask the symptoms or signs of neurological complications from surgery. Usually, neurological deficit initially manifests as non distinct sedation, confusion, or agitation, rather than gross motor deficits and could be wrongly attributed to the effects of anesthesia.

During surgery under GA there is the need to monitor the level of cerebral perfusion during arterial clamping and several different methods may be used (the measurement of stump pressure in the internal carotid artery, near-infrared spectroscopy, transcranial Doppler imaging (TCD) of the middle cerebral artery, electroencephalographic monitoring (EEG) and somatosensory-evoked potentials, or combinations of some of these techniques). All of these methods have relatively low sensitivity and specificity for detecting inadequate cerebral perfusion and intraoperative stroke, ( 15 ) with the chance of false-positive and false-negative signals. (16) Meta-analysis, (15) including 29 studies, compared the ability of different types of brain monitoring systems vs. clinical monitoring of the brain function to detect cerebral ischemia during carotid artery crossclamping under RA, and showed that a combination of stump pressure and either TCD or EEG give the best results for detecting brain ischemia during carotid artery cross clamping. For achieving the best results, EEG 
should be used with a high number of channels. (15) However, there is no established consensus as to which is the superior technique.

\section{Clinical studies: GA or RA?}

A large number of studies compare RA and GA techniques in CEA surgery patients. The primary outcome was the proportion of patients with stroke (including retinal infarction), myocardial infarction, or death.

One of the first major published studies, (17) included 803 consecutive CEAs (632 were performed under RA and 171 under GA) and showed that there was no statistically significant difference in the incidence of perioperative stroke or perioperative death between the two groups. However, the incidence of non-neurologic, nonfatal complications (eg, MI and postoperative mechanical ventilation; neck hematomas and cranial nerve injuries; and urinary retention) was significantly lower in the RA group. (17) That was partly a retrospective and prospective study and because of that, a prospective, randomized study would be needed to document this in a scientifically rigorous fashion.

A meta-analysis published in 2007 (18) included both retrospective (34 studies) and prospective (14 studies) studies and altogether included 17,028 procedures. Studies were classified as prospective randomized controlled trials (RCTs), prospective nonrandomized trials, and retrospective studies. When randomized or prospective (randomized and nonrandomized) studies were considered, there were no differences between the two types of anesthesia in regard to major outcomes, although in prospective studies the number of patients with a postoperative wound hematoma or infection was reduced with RA. (18) However, when all studies were considered together, the incidence of any new neurologic impairment, stroke, stroke and/or death, death and MI was reduced, as well as the incidence of arrhythmia, respiratory complications, and length of surgery. (18)

The disadvantage of this meta-analysis is the low number of patients included in the prospective RCTs or even in the prospective studies (randomized and nonrandomized) which was far below the minimal 
critical number needed to avoid statistical error. Mathematically, there would be no reason not to analyze retrospective studies with the same systematic technique that is usually used for prospective studies. Although, it is known that the quality of this analysis cannot be considered equivalent to the analysis of prospective studies. Actually, retrospective studies are certainly more prone to be biased for various reasons.

In an attempt to solve the dilemma which anesthetic technique is superior, the randomized GALA trial (19) recruited over 3500 patients with symptomatic or asymptomatic carotid stenosis from 95 centers between 1999 and 2007 to undergo CEA under GA or RA. Despite the enormity of the undertaking, the GALA trial results have not silenced the debate as to which anesthetic technique gives the best results. This study showed that there was no statistically significant difference in the incidence of stroke, MI, or death between randomization and 30 days after surgery. Actually, the trial was not powerful enough to detect a significant difference between GA and RA in major outcome (incidence of stroke and death). The GALA trial showed significant differences between the 2 groups in terms of shunt insertion rate (14\% RA vs. $49 \%$ GA), but shunting was used purely at the discretion of the clinicians (at least in the GA group) rather than because of detected neurological deficit. (19) Unfortunately, we do not know if the reduced shunt insertion rate had any effect on the rate of restenosis at one year (mortality rate was higher in the GA group during the first year after randomization, although this did not achieve statistical significance). More patients under GA than under RA ( $\mathrm{p}<0.001$ ) had their blood pressure manipulated up, and more patients under RA than those allocated to GA $(\mathrm{p}<0.001)$ had their blood pressure manipulated down or not manipulated at all. (19)

After the publishing of the GALA trial results, most proponents of regional anesthesia continue to criticize these results, citing the wide variability in trial methodology, which allowed any general anesthetic technique to be compared with any regional anesthesia technique, together with the changing clinical practice over the 8 years and the wide variability in practice between participating countries. On the other hand, proponents of GA have used the GALA results to validate their practice. 
The update of a Cochrane Review (20), published in 2013, included 14 randomized trials with 4596 operations. It found no statistically significant difference in the incidence of stroke, stroke and death within 30 days of surgery between the RA group and the GA group. There was a non-significant trend towards lower operative mortality with RA (RA group 0.9\%, GA 1.5\%, Peto OR 0.62, 95\% CI 0.36 to 1.07). (20)

However, neither the GALA trial nor the pooled analysis was adequately powered to reliably detect an effect on mortality.

\section{Issues beyond Stroke, Myocardial Infarction, and Death Rates}

Although there were no significant differences between the two types of anesthesia in regard to stroke, MI and death, it may be appropriate to consider other additional parameters when comparing the outcomes of these two techniques.

Thus, it analyzed the stress response that may be associated with adverse systemic effects and showed that patients under RA were exposed to higher stress levels, reflected as a rapid and significant increase in intraoperative values of cortisol and adrenocorticotropic hormone (ACTH). (21) However, in the early and late postoperative periods, the anesthetic modality no longer has an impact on surgical stress. (21)

Hemodynamic instability is very common during CEA, although patients under RA have more hypertensive episodes, while patients under GA have more hypotensive episodes during surgery. $(18,22)$ After surgery, patients in the RA group are more hemodynamically stable with a trend toward hypotension, while patients in the GA group have significantly greater hemodynamic variability and need for vasoactive medications. $(18,22)$

The majority of patients undergoing CEA are over 70 years of age. Elderly patients undergoing surgery under GA have increased rates of postoperative delirium and cognitive impairment that may result in increased morbidity and mortality, followed by delayed functional recovery, prolonged hospital stay, and consequently increased hospital 
costs. (23) Thus, employment of RA instead of GA for CEA should reduce the risk of postoperative delirium. (23)

The CEA under RA may be associated with shorter total operative time, a lower intensive care unit requirement, length of in-hospital stay and lower costs. $(17,18,24)$.

Comparing patient satisfaction, based on the use of a questionnaire consisting of 28 questions (15 preoperative and 13 postoperative questions), showed there were no differences in overall anxiety score, satisfaction score and information score, but the recovery score (measure of the patient's perception of recovery, that incorporate parameters such as postoperative nausea, pain, distress, disorientation, length of stay and return to normal activity) was significantly better in the RA group. (25) Eighty two percent of GA patients and 73\% of RA patients would choose to have the same type of anesthetic if given the choice for a second hypothetical operation (no statistical difference). (25)

\section{Conclusion}

The debate continues as to whether regional anesthesia or general anesthesia is safer. The choice of anesthetic technique is a complex decision and surgical teams should be able to offer both RA and GA. The individual choice is the ideal choice and should be determined at the discretion of the surgeon, anesthetist and patient, depending on the clinical situation and their own preferences.

\section{References}

1. Brott TG, Halperin JL, Abbara S, Bacharach JM, Barr JD, Bush RL, et al. American College of Cardiology Foundation/ American Heart Association Task Force, American Stroke Association, American Association of Neuroscience Nurses, American Association of Neurological Surgeons, American College of Radiology, American Society of Neuroradiology, Congress of Neurological Surgeons, Society of Atherosclerosis Imaging and Prevention, Society for Cardiovascular Angiography and Interventions' Society of Interventional Radiology, Society of 
NeuroInterventional Surgery, Society for VascularMedicine, Society for Vascular Surgery, American Academy of Neurology, Society of

Cardiovascular Computed Tomography. Guideline on the management of patients with extracranial carotid and vertebral artery disease: executive summary. J Neurointerv Surg 2011;3(2):100-30.

2. Liapis CD, Bell PR, Mikhailidis D, Sivenius J, Nicolaides A, Fernandes e Fernandes J, et al. ESVS guidelines. Invasive treatment for carotid stenosis: indications, techniques. Eur J Vasc Endovasc Surg 2009;37(suppl 4):1-19.

3. Brott TG, Hobson RW, Howard G, Roubin GS, Clark WM, Brooks W, et al. Stenting versus endarterectomy for treatment of carotid-artery stenosis N Engl J Med 2010;363:11-23.

4. Unic-Stojanovic D, Babic S, Neskovic V. General Versus Regional Anesthesia for Carotid Endarterectomy. J Cardiothorac Vasc Anesth 2013;27(6):1379-83.

5. Stone MC, Defreitas DJ. Process of care for carotid endarterectomy: Perioperative medical management. J Vasc Surg 2010;52:223-31.

6. Rothwell PM, Slattery J, Warlow CP. A systematic review of the risks of stroke and death due to endarterectomy for symptomatic carotid stenosis. Stroke1996;27:260-5.

7. Rothwell PM, Slattery J, Warlow CP. A systematic comparison of the risks of stroke and death due to carotid endarterectomy for symptomatic and asymptomatic stenosis. Stroke 1996;27:266-9.

8. McCarthy RJ, Walker R, McAteer P, Budd JS, Horrocks M. Patient and hospital benefits of local anesthesia for carotid endarterectomy. Eur J Vasc Endovasc Surg 2001;22:13-8.

9. McCleary AJ, Dearden NM, Dickson DH, Watson A, Gough MJ. The differing effects of regional and general anaesthesia on cerebral metabolism during carotid endarterectomy. Eur J Vasc Endovasc Surg 1996;12:173-81.

10. Trop D. Carotid endarterectomy: General is safer than regional anesthesia. J Cardiothorac Anesth 1987;1:483-8.

11. Forssell C, Takolander R, Bergqvist D, Johansson A, Persson NH. Local versus general anaesthesia in carotid surgery: A prospective, randomised study. Eur J Vasc Surg 1989;3:503-9.

12. Pandit JJ, R. Satya-Krishna R, P. Gration P. Superficial or deep cervical 
plexus block for carotid endarterectomy: A systematic review of complications. Br J Anaesth 2007;99:159-69.

13. Pandit JJ, Bree S, Dillon P, Elcock D, McLaren ID, Crider B. A comparison of superficial versus combined (superficial and deep) cervical plexus block for carotid endarterectomy: A prospective, randomized study. Anesth Analg 2000;91:781-6.

14. McCulloch TJ, Christopher L, Thompson CL. A randomized crossover comparison of the effects of propofol and sevoflurane on cerebral hemodynamics during carotid endarterectomy. Anesthesiology 2007;106:56-64.

15. Guay J, Kopp S. Cerebral monitors vs regional anesthesia to detect cerebral ischemia in patients undergoing carotid endarterectomy: a meta-analysis. Can J Anesth 2013;60:266-79.

16. Bond R, Rerkasem K, Counsell C, Salinas R, Naylor R, Warlow CP, et al. Routine or selective shunting for carotid endarterectomy (and different methods of monitoring in selective shunting). Cochrane Database Syst Rev 2002;2:CDoo0190.

17. Papavasiliou AK, Magnadottir HB, Gonda T, Franz D, Harbaugh RE. Clinical outcomes after carotid endarterectomy: Comparison of the use of regional and general anesthetics. J Neurosurg 2000;92:291-6.

18. Tangkanakul C, Counsell CE, Warlow CP. Local versus general anaesthesia in carotid endarterectomy: A systematic review of the evidence. Eur J Vasc Endovasc Surg 1997;13:491-9.

19. Lewis SC, Warlow CP, Bodenham AR, Colam B, Rothwell PM, Torgerson $D$, et al. General anaesthesia versus local anaesthesia for carotid surgery (GALA): A multicentre, randomised controlled trial. Lancet 2008;372:2132-42.

20. Vaniyapong T, Chongruksut W, Rerkasem K. Local versus general anaesthesia for carotid endarterectomy. Cochrane Database of Systematic Reviews 2013;12:CDoo0126.

21. Marrocco-Trischitta MM, Tiezzi A, Svampa MG, Bandiera G, Camilli S, Stillo F, at al. Perioperative stress response to carotid endarterectomy: The impact of anesthetic modality. J Vasc Surg 2004;39(6):1295-304.

22. Stoneham MD, Thompson JP. Arterial pressure management and carotid endarterectomy. Br J Anaesth 2009;102:442-52. 
23. Weber CF, Friedl H, Hueppe M, Hintereder G, Schmitz-Rixen T, Zwissler $\mathrm{B}$, et al. Impact of general versus local anesthesia on early postoperative cognitive dysfunction following carotid endarterectomy: GALA study subgroup analysis. World J Surg 2009;33:1526-36.

24. Gomes M, Soares MO, Dumville JC, Lewis SC, Torgerson DJ, Bodenham AR, et al. Cost-effectiveness analysis of general anaesthesia versus local anaesthesia for carotid surgery (GALA trial). Br J Surg 2010;97:1218-25.

25. McCarthy RJ. Patient Satisfaction for Carotid Endarterectomy Performed under Local Anaesthesia. Eur J Vasc Endovasc Surg 2004;27(6):654-9.

Corresponding author Dragana Unic-Stojanovic Department of Anesthesiology and Critical Care Cardiovascular Institute "Dedinje" School of Medicine, University of Belgrade Heroja Milana Tepica 1 Street, 11000 Belgrade, Serbia E-mail: dragana.unic@gmail.com

Article printed from Signa Vitae: http://www.signavitae.com URL to article: http://www.signavitae.com/2015/12/anesthesiafor-carotid-endarterectomy-where-do-we-stand-at-present/

Copyright (C) 2015 Signa Vitae. All rights reserved. 